Editorial

\title{
Dynamical Modeling, Analysis, and Control of Information Diffusion over Social Networks
}

\author{
Chenquan Gan $\mathbb{D}^{1},{ }^{1}$ Qingyi Zhu $\mathbb{D},{ }^{2}$ Wei Wang $\mathbb{D}^{3},{ }^{3}$ and Jianxin Li $\mathbb{D}^{4}$ \\ ${ }^{1}$ School of Communication and Information Engineering, Chongqing University of Posts and Telecommunications, \\ Chongqing 400065, China \\ ${ }^{2}$ School of Cyber Security and Information Law, Chongqing University of Posts and Telecommunications, \\ Chongqing 400065, China \\ ${ }^{3}$ College of Computer Science, Sichuan University, Chengdu 610065, China \\ ${ }^{4}$ School of Information Technology, Deakin University, Melbourne 3125, Australia
}

Correspondence should be addressed to Chenquan Gan; gcq2010cqu@163.com

Received 13 October 2021; Accepted 13 October 2021; Published 21 October 2021

Copyright ( $\odot 2021$ Chenquan Gan et al. This is an open access article distributed under the Creative Commons Attribution License, which permits unrestricted use, distribution, and reproduction in any medium, provided the original work is properly cited.

Information diffusion is a complex and dynamic process that involves the topological factor of social networks and the geographical and psychological factors of individuals. It has a wide range of applications, including online marketing, recommendation systems, and prevention of malware and rumors' propagation. Although lots of dynamical models describing the behaviors of information diffusion have been proposed, it is still a challenging interdisciplinary task to explain and predict the dynamics of the diffusion process in complex networks.

This special issue provides both theoreticians and practitioners with a platform to disseminate their research on the modeling, analysis, and optimization of information diffusion over social networks. All manuscripts submitted to this special issue have been reviewed via the peer-reviewing process. Based on the reviewers' comments, 16 original research articles have been accepted for publication in this well-reputed journal.

Rumor spreading is very common in information diffusion and worth studying. D. W. Huang et al. and R. Ghazzali et al. explored rumor propagation in online social networks through the optimal control approach. L. Qiu and S. Liu proposed a corrector-ignorant-spreaderweakener (C-SIW) rumor propagation model that considers the impact of variable propagation rate and perception mechanism. Y. Wu et al. studied Weibo rumor recognition by combining with communication and stacking ensemble learning.
Network structure has an important impact on information dissemination and has always received great attention. L. Zhong et al. presented an information spreading model for an activity-driven temporal network. H. Wang and L. Feng considered the information security on wireless sensor networks. Y. Wang et al. focused on the influence of network structural preference perturbation through deletion on link prediction. Q. Zhang et al. analyzed the impact of social ties on video transmission in device-to-device (D2D) communications through a stochastic approach. H. Peng et al. investigated the effect of immunization strategies on social contagions through a non-Markovian threshold model. X. Zhang and Y. Li discussed the influence of countermeasure and removable storage media on the propagation behavior of computer viruses under the fully connected networks. N. Min et al. showed the impact of student depression on the spread of public opinion in the university through a social network.

Recommendation system is an important application of information dissemination, and there are many related methods worth studying in this regard. X. Wang et al. developed a personalized recommendation approach combining the semisupervised support vector machine and active learning for collaborative filtering recommendation applications. K. Cheng et al. designed a deep-learning-based recommendation algorithm to solve the problem of data sparsity in news recommendation for social networks. M. Yin et al. proposed a representation method that 
combines different features with word vectors for social opinion mining. T. Wang et al. established a configurable semantic-based automatic conceptual model transformation methodology to improve the efficiency of the building process. Q. Shi et al. introduced a method that employs the depth map to perform extrinsic calibration automatically.

\section{Conflicts of Interest}

The editors declare that they have no conflicts of interest regarding the publication of this special issue.

\section{Acknowledgments}

The editors are very grateful to all of the authors for their outstanding contributions in this field and the reviewers for their valuable comments on the evaluation of the papers during the reviewing process.

Chenquan Gan Qingyi Zhu Wei Wang Jianxin Li 